\title{
Noncoding RNAs: Different roles in tumorigenesis
}

\author{
LIN Mei ${ }^{1}$, WU Jing ${ }^{2} \&$ SHAN Ge ${ }^{1 *}$ \\ ${ }^{1}$ School of Life Sciences, University of Science and Technology of China, Hefei 230026, China; \\ ${ }^{2}$ School of Life Science and Technology, Huazhong University of Science and Technology, Wuhan 430074, China
}

Received August 9, 2011; accepted October 8, 2011

\begin{abstract}
A major portion of the mammalian genome is transcribed to produce large numbers of noncoding RNAs (ncRNAs). During the past decade, the discovery of small RNAs, including the microRNAs (miRNA) and small interfering RNAs (siRNA), has led to important advances in biology. The breadth of the ncRNA field of study has substantially expanded and many recent results have revealed a range of functions that can be attributed to the miRNAs and other ncRNAs. For example, H19 RNA, HOTAIR RNA, transcribed ultraconserved regions (T-UCRs), natural antisense RNA, transfer RNA and mitochondrial noncoding RNA have been suggested to play important roles in cancers and other diseases as well as in diverse cellular processes. In this review, we focus on the current status of several classes of ncRNAs associated with cancer with the emphasis on those that are not microRNAs.
\end{abstract}

noncoding RNA, cancer, microRNA

Citation: Lin M, Wu J, Shan G. Noncoding RNAs: Different roles in tumorigenesis. Chin Sci Bull, 2012, 57: 959-965, doi: 10.1007/s11434-011-4917-x

Mounting evidence has revealed that a major portion of the mammalian genome is transcribed to produce large numbers of noncoding RNAs (ncRNAs), that is, RNAs that either do not have an open reading frame or RNAs that have a short poorly conserved open reading frame and do not code for a protein $[1,2]$. During the past decade, the discovery of small RNAs including the microRNAs (miRNAs) and small interfering RNAs (siRNAs) has led to major advances in biology. After miRNAs were first connected to cancer pathogenesis [3], accumulating data have pointed to a central regulatory role for miRNAs in the initiation and progression of most of the cancers that have been analyzed so far. Recently, the breadth of the ncRNA field of study has substantially expanded. A series of studies have revealed the essential roles of many ncRNAs in diverse cellular processes including cancer; these ncRNAs include H19 RNA, HOTAIR RNA, transcribed ultraconserved regions (T-UCRs), natural antisense RNA, transfer RNA and mitochondrial noncoding RNA (Figure 1). In this review, we focus on the current status of the research into the several classes of ncRNAs

*Corresponding author (email: shange@ustc.edu.cn) associated with cancer.

\section{MicroRNAs and cancer}

MicroRNAs (miRNAs), a category of small ncRNA molecules with lengths of 18-25 nucleotides, were discovered recently. MiRNAs act as post-transcriptional regulators of genes by sequence-specific binding to the $3^{\prime}$ untranslated regions (3' UTRs) of target mRNAs. Release 17 (April 2011) of the miRBase database (http://microrna.sanger.ac.uk/) catalogues 1424 human miRNAs. Over $60 \%$ of human proteincoding genes have been suggested to be under selective pressure to maintain their pairing to miRNAs [4]. Depending on the degree of sequence complimentary between the miRNA and 3' UTR, the pairing might result in inhibition of translation and/or degradation of the target mRNA [5,6].

Several miRNAs that previously were described as suppressors have been found to be either deleted or mutated in various human malignancies. Calin et al. [3] first reported that $m i R-15 a$ and $m i R-16-1$, located in a cluster on chromosome $13 q 14$, a frequently deleted region in B cell chronic 


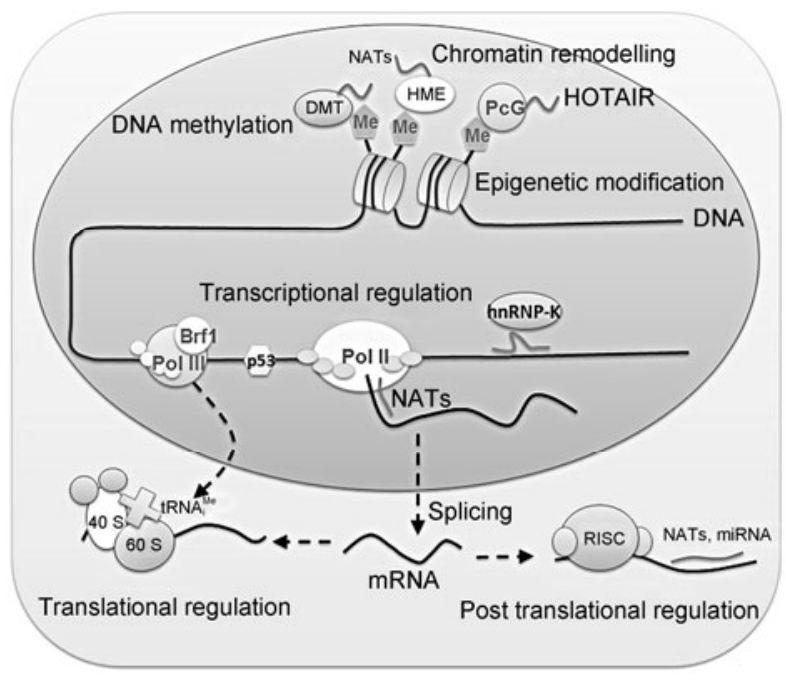

Figure 1 Summary of ncRNAs involved in tumorigenesis and cancer biology. The roles of each of the ncRNA are described in the corresponding sections of the review.

lymphocytic leukemia (CLL), could function as tumor suppressor genes. This finding prompted the mapping of all miRNA genes to chromosomal locations. The mapping revealed that a significant fraction of miRNAs mapped to cancer-associated genomic regions in human [7] and mouse [8], suggesting a connection between the deregulation of miRNAs and tumorigenesis accompanied by chromosomal aberrations such as deletions and amplifications.

The loss of members of the let-7 family of miRNAs resulted in the constitutive overexpression of the ras oncogene [9]. It was found that $m i R-15 a$ and $m i R-16-1$ target the $B C L 2$ oncogene that inhibits apoptosis [3]. It is clear that the loss of the miRNAs could contribute to malignant transformation through the dysfunction of cellular oncogenes.

However, in various human tumors, the overexpression of miRNAs has been observed and the evidence indicated that some of these miRNAs could function as oncogenes. The two miRNAs that were first found to be overexpressed in cancers were the miR-155 and miR-17-92 clusters [10,11]. Interestingly, in solid cancers of epithelial origin and in leukemias and lymphomas, $m i R-155$ was highly expressed and acted as an oncogene. However, in endocrine tumors it was significantly down-regulated and possibly had suppressive functions [12]. These studies suggest that the roles of miRNAs in cancers are tissue and tumor specific.

Various biological processes associated with cancer are regulated by microRNAs; they include transcription, cellcycle regulation, apoptosis, angiogenesis, invasion and metastasis [13]. Numerous miRNA-target gene pairs have been reported to be involved with tumorigenesis (for a review, see [14]).

Because miRNA expression profiles reflect tumor origin, stage and other pathological variables, the profiles could be used as diagnostic or prognostic tools. Furthermore, in tumors in which miRNAs are either lost or overexpressed, corresponding miRNAs or anti-miRNAs [15] might be considered as drugs that could induce apoptosis and/or cell cycle arrest in cancer cells depending on their deregulation role for growth and survival. Here we have briefly reviewed the relationship between miRNAs and cancer; however, there are many reports and reviews that discuss this subject and readers are referred to the related references for more detailed information $[16,17]$.

\section{Roles of other noncoding RNAs in cancer}

While recent studies strongly support the role of microRNAs in the pathogenesis of the majority of cancers studied so far, recent investigations into the functions of other ncRNAs have indicated that they also play essential roles in the pathogenesis of cancers. These results add a new layer of complexity to the molecular architecture of human cancers. Here we briefly review several types of ncRNAs that drive cancer development and progression through different mechanisms in human cancers.

\subsection{Large intergenic noncoding RNAs (lincRNAs)}

There is growing recognition that mammalian cells produce many thousands of large intergenic transcripts [18]. To date, about a dozen functionally well-characterized large intergenic noncoding RNAs (lincRNAs) with transcript sizes ranging from 2.3 to 17.2 kilonucleotide (knt) have been reported in mammals $[19,20]$. These lincRNAs have distinctive biological roles in diverse molecular mechanisms that include imprinting (e.g. H19 RNA), trans-acting gene regulation (e.g. HOTAIR RNA), mediating global gene repression in the p53 response (e.g. lincRNA-p21), functioning in $\mathrm{X}$-chromosome inactivation (e.g. Xist) [20] and regulating nuclear import (e.g. Nron) [21]. Recent studies suggest that some of the lincRNAs, for example H19 and HOTAIR, are involved in cancer progression and metastasis.

(i) H19 RNA. H19 is a maternally imprinted gene located on chromosome $11 \mathrm{p} 15.5$. It transcribes into a spliced 2.5-knt polyadenylated long ncRNA (H19 RNA). Postnatally, its expression is shut off in most tissues; it is reactivated during adult tissue regeneration and tumorigenesis $[22,23]$. The imprinted cluster loci have been implicated in a variety of disorders and cancer predispositions for both pediatric and adult tumors. This observation implied that H19 was a potential tumor suppressor. Studies by Hao and Crenshaw [24] demonstrated that the induced expression of a transfected copy of H19 RNA suppressed cellular proliferation and tumorigenesis in certain tumor cell lines. This further placed the $H 19$ as a candidate gene with some kind of role in tumorigenesis. Later studies found that H19 expression was reduced or extinguished in a proportion of Wilms' tumors and embryonal rhabdomyosarcoma [25,26]. These data show that $H 19$ can act as a tumor suppressor gene. 
Numerous reports have linked H19 expression and carcinogenesis, providing evidence for $\mathrm{H} 19$ as an oncogene $[23,27]$ and recent studies have shown that altered H19 expression is associated with different stages of tumorigenesis, such as cell de-differentiation [28], blood vessel development [29] and tumor metastasis [30].

Even though many related studies have identified $H 19$ RNA that are aberrantly expressed in cancers, how H19 fulfills its tumor suppressing or oncogenic tasks remains poorly understood. Berteaux and Lottin [31] found that $H 19$ overexpression had a significant impact on breast cancer cell proliferation; H19 expression accelerated S-phase entry and cell cycle progression by controlling the direct and specific binding of transcription factor E2F1 to its promoter. A miRNA ( $m i R-675)$ has recently been profiled in exon 1 of the human $H 19$ genes, suggesting that H19 RNA may have two different functions: one as the spliced full-length transcript and the other as the miR-675 precursor [32]. This duality could explain the conflicting functions (either as a growth suppressor or as a growth enhancer) that have been ascribed to $H 19$ in the control of tumor growth.

$I G F-2$ is an oncogene which was identified as an oppositely imprinted (paternally imprinted) gene to $H 19$ in the same imprinting cluster [33]. H19 and IGF-2 have similar expression patterns in the same tissues at the same developmental stages during embryogenesis [34]. Losses of imprinting of the IGF2 gene and inactivation of the $H 19$ gene have been implicated in the pathogenesis of embryonal tumors [35]. This pattern of gene expression suggests that these two genes may be co-regulated. In addition, H19 RNA was reported to be involved in the repression of the $I G F-2$ oncogene by affecting either its transcription [36] or its translation [37].

Different research groups have reported several different elements that regulate $H 19$ expression in tumors. Boutros et al. [23] identified c-Myc that directly binds to the H19 promoter and sustantially up-regulates the transcription of the maternal $H 19$ allele by recruiting histone acetyltransferase (HAT). Their results have further indicated that up-regulation of $\mathrm{H} 19$ by Myc contributes significantly to the tumorigenic phenotype of breast and lung cancer cells. In breast cancer, $H 19$ was reported to be a target gene for hepatocyte growth factor (HGF) [38]. These results suggest the possibility of using the $H 19$ promoter in gene therapy to drive the expression of cytotoxic genes in tumorigenic cells [39].

(ii) HOTAIR. Rinn and Kertesz [40] have characterized a 2158 nucleotide lincRNA, termed HOTAIR (for HOX antisense intergenic RNA), and made the surprising discovery that this ncRNA acts in trans to regulate a $H O X$ gene cluster on a completely different chromosome, not the $H O X$ gene cluster that encodes the ncRNA itself. Mammalian homeobox (Hox) genes (HOXA-D), which establish the body plan during early development, are clustered at four chromosomal loci. HOTAIR, which is transcribed from the HOX C locus, can target Polycomb repressive complex 2 (PRC2) and other chromatin regulators to the HOX D locus and potentially many other genomic loci [40-42]. Rinn and Kertesz [40] have profiled expression patterns of the transcriptional landscape of these loci and identified ncRNAs corresponding to chromatin regions with different epigenetic modifications, suggesting the mechanism might be epigenetic; however, this still remains an open question. Nevertheless, this is the first example of an RNA expressed on one chromosome influencing transcription on another chromosome. These findings reveal a new mechanism whereby ncRNAs can determine the silencing of distant chromosome regions that might have important implications for both disease and development.

Recently, Gupta and Shah [41] reported that HOTAIR is systematically dysregulated in breast carcinoma and, notably, that HOTAIR regulates metastatic progression. They found that HOTAIR recruits the PRC2 complex to specific target genes genome-wide, leading to alteration of histone H3 lysine 27 methylation, epigenetic silencing of metastasis suppressor genes (such as JAM2, PCDH1O and PCDHB5), and inducing positive regulator genes of metastasis (such as ABL2, SNAIL, and laminins). HOTAIR-altered gene expression was reversed after PRC2 depletion.

The interdependence between HOTAIR and PRC2 may indicate potential therapeutic applications. Tumors that are sensitive to inhibitors of PRC2 could be identified by monitoring the increased level of HOTAIR [43]. Conversely, suppression of endogenous HOTAIR or inhibition of the HOTAIR-PRC2 interaction may provide potential therapeutic targets to tumors that overexpress Polycomb proteins.

(iii) LincRNA-p21. In investigations into the potential biological roles of lincRNAs, several lincRNAs were identified to be regulated by $p 53$. Huarte et al. [44] have demonstrated that numerous lincRNAs are important components in the p53-dependent transcriptional pathway. Of these, lin$c R N A-p 21$ functions as a direct $p 53$ transcriptional target in response to DNA damage; it is required for $p 53$-dependent apoptotic responses to DNA damage. LincRNA-p21 acts by modulating nuclear ribonucleoprotein $\mathrm{K}$ (hnRNP-K) localization through its interaction with hnRNP-K.

Various hypothetical mechanisms by which lincRNA$p 21$ could contribute to repression at specific loci have been suggested. They include: (1) lincRNA-p21 might direct a protein complex to specific loci by Crick-Watson base pairing; (2) lincRNA-p21 might act by forming DNA-DNARNA triple-helical structures; or (3) lincRNA-p21 might alter the binding specificity of DNA-binding proteins to influence their target preference. The precise mechanism needs further confirmation.

Because $p 53$ is a well-known tumor suppressor gene, it is clear that lincRNA-p21 and several other lincRNAs function in an important pathway for cancer. It is tempting to speculate that other lincRNAs may also play important roles in numerous other tumor suppressor or oncogenic pathways, suggesting that the lincRNAs represent an unknown para- 
digm in cellular transformation and metastasis. It is important to determine whether lincRNA genes can serve as tumor suppressor genes or oncogenes in future studies.

\subsection{Transcribed ultraconserved regions (T-UCRs)}

Evolutionarily conserved sequences within or adjoining orthologous genes often serve as critical cis-regulatory regions. Recent studies have identified long, noncoding genomic regions that are perfectly conserved in human, mouse and rat genomes; these regions are termed ultra-conserved regions (UCRs). UCRs represent a group of sequences 200$779 \mathrm{bp}$ in length that were first discovered by Bejerano in $2004[45,46]$. UCRs are frequently located at fragile sites and genomic regions involved in cancers. A large fraction of the genomic UCRs encode a particular set of ncRNAs called transcribed ultraconserved regions (T-UCRs) that exhibit altered expression in human cancers [47].

Calin et al. [47] demonstrated that a new class of UCRs was significantly altered at both the DNA and RNA levels in adult chronic lymphocytic leukemias (CLL), colorectal and hepatocellular carcinomas. In colon cancer, $u c .73 \mathrm{~A}$, one of the most statistically up-regulated transcribed-UCRs (TUCRs), was found to induce cell proliferation by reducing apoptosis, suggesting its function as an oncogene.

Scaruffi et al. [48] suggested that deregulation of the microRNA/T-UCR network may play an important role in the pathogenesis of neuroblastoma and tumor-associated T-UCRs in CLL were found to be regulated by certain miRNAs [47]. These results led to the hypothesis that T-UCRs along with miRNAs may define signatures associated with the diagnosis, prognosis and treatment of tumors. The roles of T-UCRs in cancer cells remain to be defined.

\subsection{Natural antisense RNA}

Natural antisense transcripts (NATs), also called antisense RNAs, are a group of RNAs that contain sequences that are complementary to other endogenous transcripts. Both sense and antisense RNAs can either encode proteins or be noncoding transcripts; however, the most prominent product of antisense transcription in the mammalian genome is a noncoding antisense RNA partner of a protein-coding transcript [49]. To date, numerous NATs with repressive functions have been described in different organisms. They can be transcribed in cis from opposing DNA strands at the same genomic loci (cis-NATs), or in trans from separate loci (trans-NATs). Most of the studies in the past few years have focused on cis-NATs.

Earlier studies identified a certain numbers of sense-antisense transcripts exhibing interactions that were associated with cancer. For example, $B c l-2$ antisense RNA $I g H$ deregulates $B c l$-2 gene expression in human follicular lymphoma cell lines [50] and the NAT of EPR-1 (effecter cell protease receptor-1) down-regulates survivin expression, thus, func- tioning as an inhibitor of apoptosis in a human colon cancer cell line [51]. Although many NATs have been identified recently, our understanding of how antisense transcripts regulate gene expression in human cells remains mostly incomplete. Recent functional validation investigations have indicated that antisense transcripts are not a uniform group of regulatory RNAs but instead belong to multiple categories that have some common features. Pioneering studies in several eukaryotic systems have identified a number of proposed mechanisms for the antisense-mediated regulation of sense mRNA gene expression [52]. The proposed mechanisms have been categorized into four main groups: mechanisms related to transcription, RNA-DNA interactions (such as genomic imprinting, alteration of DNA methylation and chromatin modification), nuclear RNA duplex formation, and cytoplasmic RNA duplex formation (such as the formation of endogenous siRNAs and masking miRNA-binding sites). For a review of these mechanisms, see [53].

The growing list of validated sense-antisense transcript pairs includes many important developmental genes as well as genes known to be involved in various human disorders, including cancer. It is well known that suppression of transcription is usually brought about by DNA and chromatin modification at the promoter region of the sense strand. Previous studies have reported a few examples of antisense transcripts that are implicated in cancer via their role in altering DNA and chromatin modification. p15 is a welldocumented tumor suppressor gene that is frequently genetically deleted or epigenetically silenced in a wide variety of tumors including leukemia, melanomas, gliomas and lung cancers. Recently, Yu et al. [54] identified a p15 antisense RNA ( $p 15 A S$ RNA), which can induce epigenetic silencing of $p 15$. This study demonstrated that the antisense RNA could silence its sense gene at the transcriptional level by affecting DNA methylation and histone modifications. This result was confirmed by Morris [55] who found that $p 21 A S$ RNA directed the trimethylation of histone $\mathrm{H} 3$ at lysine 27 (H3K27me3) in the $p 21$ sense promoter region, resulting in the suppression of $p 21$ gene expression. In addition, $p 53$ as a tumor suppressor gene plays an important role in the prevention of human cancer and its mutations result in human tumors. Recent studies have revealed that Wrap53, a natural antisense transcript of $p 53$, plays an important role in the regulation of $p 53 \mathrm{mRNA}$ and in the induction of the p53 protein by targeting the $5^{\prime}$ UTR of $p 53$ mRNA. Unlike the $p 15$ and $p 21$ antisense RNAs, Wrap53 antisense and $p 53$ are co-expressed in cells [56].

NATs associated with genomic imprinting were identified in several reports. A region on chromosome 6 that contains three maternally imprinted protein-coding genes ( $I g f 2 r$, Slc22a2 and Slc22a3) has been shown to be controlled by a paternally expressed noncoding antisense $\operatorname{Ig} 2 \mathrm{2} r$ RNA (Air) [57]. Air is a $108-\mathrm{kb}$ unspliced and repeat-rich transcript which only overlaps in an antisense orientation with $I g f 2 r$ and not with Slc22a2 or Slc22a3 [58]. However, the expres- 
sion of Air can silence all three protein-coding genes in the imprinted cluster [59].

Significantly, the development of tools to identify antisense transcripts that are associated with cancer loci should provide a better insight into the functions of different antisense transcripts and the molecular etiology associated with many important human cancers. Moreover, the ability of NATs to regulate the expression of sense transcripts could provide numerous new potential drug targets in the emerging field of functional antisense transcripts.

\subsection{Transfer RNA}

The increased expression of RNA polymerase (pol) III transcription factors and of pol III products (such as tRNA and 5S rRNA) has often been observed in transformed and tumor cells in, for example, ovarian and cervical carcinomas and breast cancer [60-62]. However, whether pol III is a causative factor in cancer is unclear because recurrent mutations in pol III subunits or the associated transcription factors have not been found in tumors.

tRNAs are one large class of pol III noncoding transcripts. Earlier research showed that the tRNA methyltransferase Misu was overexpressed in breast and colon cancers, and that Misu RNAi (RNA interference) inhibited growth of carcinomas [63], supporting the conclusion that tRNA metabolism can have a strong influence on oncogenic transformation.

Recently, White et al. [64] reported that elevated tRNA production can have a causal role in oncogenic transformation. They demonstrated that the phenotypic effects of pol III transcription factor Brf1 induction was mimicked by elevated levels of a pol III product $\mathrm{RNA}_{\mathrm{i}}{ }^{\mathrm{Met}}$, the initiator tRNA $^{\text {Met }}$. Moreover, a modest overexpression of $\mathrm{tRNA}_{\mathrm{i}}{ }^{\mathrm{Met}}$ was found to significantly stimulate translation, suggesting the activation of tRNA expression that was often observed in transformed cells might have functional consequences for the development of cancer [64]. White et al. created stably transfected lines of immortalized mouse embryonic fibroblasts carrying cDNA encoding tRNA ${ }^{\text {Met }}$ that expressed elevate levels of cyclin D1 and c-Myc proteins without corresponding changes in the levels of their mRNA. Mei et al. [65] microinjected tRNA into cells and found that this led to significant inhibition of cytochrome $c$-induced apoptosis. Therefore, the tRNA levels in cells may be relevant for the translation of the important genes required for the tumorigenic process and may play a central role in cancer development and progression. The precise mechanism and possible therapeutic applications of tRNA in cancer need to be explored further.

\subsection{Noncoding mitochondrial RNAs}

The mammalian mitochondrial genome is a double-stranded circular DNA about 16500 nucleotides long [66]. It encodes the $12 \mathrm{~S}$ and $16 \mathrm{~S}$ ribosomal RNAs, 22 tRNAs and 13 polypeptides [67]. Besides these coding regions, the mitochondrial DNA (mtDNA) contains an approximately $1-\mathrm{kb}$ noncoding region called the D-loop region that contains the origin of $\mathrm{H}$-strand replication and the promoters for transcription of the H-strand and L-strand templates [68].

Several models that link mitochondrial dysfunction to cancer have been proposed. However, only a few of these models discuss the role of noncoding mitochondrial RNA (ncmtRNA) in cancer, and the precise mechanism of their involvement is yet to be defined.

mtDNA mutations have been linked to a broad spectrum of malignancies. Mutations have been described in both rRNAs, in all 22 tRNAs, in all 13 of the mtDNA-encoded subunits of the respiratory chain complexes and in the mitochondrial D-loop regions [69]. Interestingly, about half of the disease-related mutations (237 in total 480) are located within the ncmtRNA genes (see http://www.mitomap.org/ MITOMAP) and, given that these sequences comprise only $10 \%$ of the mitochondrial genome, this result indicates a possible role for the ncmtRNAs in various diseases including cancer [70].

Mei et al. [65] have reported that when mitochondrial tRNAs along with cytosolic tRNAs bind to cytochrome $c$ they impair the interaction of cytochrome $c$ with Apaf-1, a caspase activator, and prevent the formation of the apoptosome. This finding raised the possibility that mitochondrial tRNAs may play a role in determining cellular responsiveness to apoptotic stimuli.

In mouse cells, Villegas et al. [71,72] identified the presence of a mitochondrial RNA (mtRNA) that contained an inverted repeat (IR) of 121 nucleotides that covalently linked to the $5^{\prime}$ end of the mitochondrial 16S RNA. Later the same workers demonstrated that human cells contain a transcript of $2374 \mathrm{nt}$ with similar structural features to the mouse mtRNA. This transcript contained a stem-loop structure with an 820-bp double-stranded region and a 40-nt loop, and that it was expressed in human proliferating cells but not in resting cells, suggesting that this ncmtRNA may play a role in cell proliferation [73]. Recently, Burzio et al. [74] reported that in addition to this transcript, normal human proliferating cells in culture expressed two ncmtRNA transcripts, sense ncmtRNA (SncmtRNA) and antisense ncmtRNA (ASncmtRNA). The expression of SncmtRNA and the down-regulation of the ASncmtRNAs was observed in 15 different tumor cell lines and in tumor cells present in 273 cancer biopsies corresponding to 17 different cancer types. The correlation of SncmtRNA and the replicative state of the cells suggests that SncmtRNA may play a role in regulating cell cycles. However, how this molecule might participate in the regulation of cell cycles remains unknown. Our understanding of ASncmtRNAs is even lower; the down-regulation of ASncmtRNAs in tumor cells suggests its possible role as a unique mitochondria-encoded tumor suppressor [74]. 


\subsection{Other ncRNAs}

Many other ncRNAs including Alu RNA [75], Y RNA [76], and small nucleolar RNA [77], show abnormal expression patterns in cancerous tissues. Their roles and possible mechanisms are not reviewed here; however, some details about them are described in the references.

\section{Conclusion}

An understanding of ncRNA is crucial to the early detection and better diagnosis of cancer, for a more accurate description of the tumor type, and for a more effective therapy. Potentially, an attractive model of cancer treatments is either to activate tumor-suppressor genes or to silence oncogenes by programming specific noncoding RNAs.

The authors thank to members of the Ge Shan lab for discussion. This work was supported by the National Basic Research Program of China (2011CBA01103), National Natural Science Foundation of China (31071132), and the Fundamental Research Funds for the Central Universities (HUST: 2010ZD022).

1 Carninci P, Kasukawa T, Hayashizaki Y, et al. The transcriptional landscape of the mammalian genome. Science, 2005, 309: 15591563

2 Kapranov P, Willingham A T, Gingeras T R. Genome-wide transcription and the implications for genomic organization. Nat Rev Genet, 2007, 8: 413-423

3 Calin G A, Dumitru C D, Shimizu M, et al. Frequent deletions and down-regulation of micro-RNA genes miR15 and miR16 at $13 \mathrm{q} 14$ in chronic lymphocytic leukemia. Proc Natl Acad Sci USA, 2002, 99: 15524-15529

4 Friedman R C, Farh K K, Burge C B, et al. Most mammalian mRNAs are conserved targets of microRNAs. Genome Res, 2009, 19: 92-105

5 Kawasaki H, Taira K. MicroRNA-196 inhibits HOXB8 expression in myeloid differentiation of HL60 cells. Nucleic Acids Symp Ser, 2004, 48: 211-212

6 Eulalio A, Huntzinger E, Nishihara T. Deadenylation is a widespread effect of miRNA regulation. RNA, 2009, 15: 21-32

7 Calin G A, Sevignani C. Human microRNA genes are frequently located at fragile sites and genomic regions involved in cancers. Proc Natl Acad Sci USA, 2004, 101: 2999-3004

8 Sevignani C, Calin G A. MicroRNA genes are frequently located near mouse cancer susceptibility loci. Proc Natl Acad Sci USA, 2007, 104: 8017-8022

9 Johnson S M, Grosshans H. RAS is regulated by the let-7 microRNA family. Cell, 2005, 120: 635-647

10 Eis P S, Tam W. Accumulation of miR-155 and BIC RNA in human B cell lymphomas. Proc Natl Acad Sci USA, 2005, 102: 3627-3632

11 Ota A, Tagawa H, Karnan S, et al. Identification and characterization of a novel gene, C13orf 25, as a target for 13q31-q32 amplification in malignant lymphoma. Cancer Res, 2004, 64: 3087-3095

12 Calin G A, Croce C M. MicroRNA signatures in human cancers. Nat Rev Cancer, 2006, 6: 857-866

13 Lee Y S, Dutta A. MicroRNAs in cancer. Annu Rev Pathol Mech Dis, 2009, 4: 199-227

14 Ryan B M, Robles A I, Harris C C. Genetic variation in microRNA networks: The implications for cancer research. Nat Rev Cancer, 2010, 10: 389-402

15 Krützfeldt J, Rajewsky N. Silencing of microRNAs in vivo with "antagomirs". Nature, 2005, 438: 685-689
16 Ryan B M, Robles A I, Harris C C. Genetic variation in microRNA networks: The implications for cancer research. Nat Rev Cancer, 2010, 10: 389-402

17 Kwak P B, Iwasaki S, Tomari Y. The microRNA pathway and cancer. Cancer Sci, 2010, 101: 2309-2315

18 Bertone P, Stolc V, Royce T E. Global identification of human transcribed sequences with genome tiling arrays. Science, 2004, 24: 2242-2246

19 Brannan C I, Dees E C. The product of the $H 19$ gene may function as an RNA. Mol Cell Biol, 1990, 10: 28-36

20 Brown C J, Ballabio A. A gene from the region of the human X inactivation centre is expressed exclusively from the inactive $\mathrm{X}$ chromosome. Nature, 1991, 349: 38-44

21 Willingham A T, Orth A P. A strategy for probing the function of noncoding RNAs finds a repressor of NFAT. Science, 2005, 309: $1570-1573$

22 Brunkow M E, Tilghman S M. Ectopic expression of the $\mathrm{H} 19$ gene in mice causes prenatal lethality. Genes Dev, 1991, 5: 1092-1101

23 Barsyte-Lovejoy D, Lau S K, Boutros P C. The $c-M y c$ oncogene directly induces the $H 19$ noncoding RNA by allele-specific binding to potentiate tumorigenesis. Cancer Res, 2006, 66: 5330-5337

24 Hao Y, Crenshaw T. Tumour-suppressor activity of H19 RNA. Nature, 1993, 365: 764-767

25 Steenman J C, Rainier S. Loss of imprinting of IGF2 is linked to reduced expression and abnormal methylation of $\mathrm{H} 19$ in Wilms' tumour. Nat Genet, 1994, 7: 433-439

26 Moulton T, Crenshaw T. Epigenetic lesions at the H19 locus in Wilms' tumour patients. Nat Genet, 1994, 7: 440-447

27 Matouk I J, DeGroot N. The H19 noncoding RNA is essential for human tumor growth. PLoS ONE, 2007, 2: e845

28 Scott R E, Gao S. De-differentiation-derived mesenchymal stem cells demonstrate selective repression in $\mathrm{H} 19$ bioregulatory RNA gene expression. Differentiation, 2005, 73: 294-302

29 Ayesh S, Matouk I. Possible physiological role of H19 RNA. Mol Carcinog, 2002, 35: 63-74

30 Yang J, Mani S A. Twist, a master regulator of morphogenesis, plays an essential role in tumor metastasis. Cell, 2004, 117: 927-939

31 Berteaux N, Lottin S. H19 mRNA-like noncoding RNA promotes breast cancer cell proliferation through positive control by E2F1. J Biol Chem, 2005, 280: 29625-29636

32 Cai X Z, Cullen B R. The imprinted $H 19$ noncoding RNA is a primary microRNA precursor. RNA, 2007, 13: 313-316

33 Leighton P A, Saam J R. An enhancer deletion affects both H19 and Igf2 expression. Genes Dev, 1995, 9: 2079-2089

34 Ekstrom T J, Cui H. Promoter-specific IGF2 imprinting status and its plasticity during human liver development. Development, 1995, 121: 309-316

35 DeBaun M R, Niemitz E L. Epigenetic alterations of H19 and LIT1 distinguish patients with Beckwith-Wiedemann syndrome with cancer and birth defects. Am J Hum Genet, 2002, 70: 604-611

36 Wilkin F, Paquette J. H19 sense and antisense transgenes modify insulinlike growth factor-II mRNA levels. Eur J Biochem, 2000, 267: 40204027

37 Li Y M, Franklin G. The $H 19$ transcript is associated with polysomes and may regulate IGF2 expression intrans. J Biol Chem, 1998, 273: 28247-28252

38 Toillon R A, Descamps S, Adriaenssens E. Hepatocyte growth factor enhances CXCR4 expression favoring breast cancer cell invasiveness. Exp Cell Res, 2005, 310: 176-185

39 Banet G, Bibi O. Characterization of human and mouse $\mathrm{H} 19$ regulatory sequences. Mol Biol Rep, 2000, 27: 157-165

40 Rinn J L, Kertesz M. Functional demarcation of active and silent chromatin domains in human $\mathrm{HOX}$ loci by noncoding RNAs. Cell, 2007, 129: 1311-1323

41 Gupta R A, Shah N. Long noncoding RNA HOTAIR reprograms chromatin state to promote cancer metastasis. Nature, 2010, 464: 1071-1076

42 Tsai M C, Manor O, Wan Y, et al. Long noncoding RNA as modular scaffold of histone modification complexes. Science, 2010, 6: 689-693 
43 Tan J, Yang X, Zhuang L, et al. Pharmacologic disruption of Polycomb-repressive complex 2-mediated gene repression selectively induces apoptosis in cancer cells. Genes Dev, 2007, 21: 1050-1063

44 Huarte M, Guttman M, Feldser D, et al. A large intergenic noncoding RNA induced by p53 mediates global gene repression in the p53 response. Cell, 2010, 142: 409-419

45 Bejerano G. Ultraconserved elements in the human genome. Science, 2004, 304: 1321-1325

46 Bejerano G. Into the heart of darkness: Large-scale clustering of human noncoding DNA. Bioinformatics, 2004, 20: i40-i48

47 Calin G A, Liu C G, Ferracin M, et al. Ultraconserved regions encoding ncRNAs are altered in human leukemias and carcinomas. Cancer Cell, 2007, 12: 215-229

48 Scaruffi P, Stigliani S, Moretti S, et al. Transcribed-ultra conserved region expression is associated with outcome in high-risk neuroblastoma. BMC Cancer, 2009, 9: 441

49 Katayama S, Tomaru Y. Antisense transcription in the mammalian transcriptome. Science, 2005, 309: 1564-1566

50 Capaccioli S, Quattrone A, Schiavone N, et al. A $b c l-2 / I g H$ antisense transcript deregulates $b c l-2$ gene expression in human follicular lymphoma $\mathrm{t}(14 ; 18)$ cell lines. Oncogene, 1996, 13: 105-115

51 Yamamoto T, Manome Y, Nakamura M, et al. Downregulation of survivin expression by induction of the effector cell protease receptor-1 reduces tumor growth potential and results in an increased sensitivity to anticancer agents in human colon cancer. Eur J Cancer, 2002, 38: 2316-2324

52 Cui I, Cui H. Antisense RNAs and epigenetic regulation. Epigenomics, 2010, 2: 139-150

53 Faghihi M A. Regulatory roles of natural antisense transcripts. Nat Rev Mol Cell Biol, 2009, 10: 637-643

$54 \mathrm{Yu} \mathrm{W}$, Gius D, Onyango P, et al. Epigenetic silencing of tumour suppressor gene $p 15$ by its antisense RNA. Nature, 2008, 451: 202206

55 Morris $\mathrm{K} \mathrm{V}$. Bidirectional transcription directs both transcriptional gene activation and suppression in human cells. PLoS Genet, 2008, 4: e1000258

56 Mahmoudi S. Wrap53, a natural p53 antisense transcript required for p53 induction upon DNA damage. Mol Cell, 2009, 33: 462-471

57 Sleutels F, Zwart R, Barlow D P. The noncoding Air RNA is required for silencing autosomal imprinted genes. Nature, 2002, 415: 810-813

58 Zwart R, Sleutels F, Wutz A, et al. Bidirectional action of the $\operatorname{Ig} 22 r$ imprint control element on upstream and downstream imprinted genes. Genes Dev, 2001, 15: 2361-2366

59 Lyle R, Watanabe D, te Vruchte D, et al. The imprinted antisense RNA at the $\operatorname{Ig} f 2 r$ locus overlaps but does not imprint Mas1. Nat Genet, 2000, 25: 19-21

60 Winter A G, Sourvinos G, Allison S J, et al. RNA polymerase III transcription TFIIIC2 is overexpressed in ovarian tumours. Proc Natl
Acad Sci USA, 2000, 97: 12619-12624

61 Daly N L, Arvanitis D A, Fairley J A, et al. Deregulation of RNA polymerase III transcription in cervical epithelium in response to high-risk human papillomavirus. Oncogene, 2005, 24: 880-888

62 Pavon-Eternod M, Gomes S, Geslain R, et al. tRNA over-expression in breast cancer and functional consequences. Nucleic Acids Res, 2009, 37: 7268-7280

63 Frye M, Watt F M. The RNA methyltransferase Misu (NSun2) mediates myc-induced proliferation and is upregulated in tumors. Curr Biol, 2006, 16: 971-981

64 Marshall L, Kenneth N S, White R J. Elevated tRNA ${ }_{i}^{\text {Met }}$ synthesis can drive cell proliferation and oncogenic transformation. Cell, 2008, 133: 78-89

65 Mei Y, Yong J, Liu H, et al. tRNA binds to cytochrome $c$ and inhibits caspase activation. Mol Cell, 2010, 37: 668-678

66 Anderson S, Bankier A T, Barrell B G, et al. Sequence and organization of the human mitochondrial genome. Nature, 1981, 290: 457-465

67 Fernández-Silva P, Enriquez J A, Montoya J. Replication and transcription of mammalian mitochondrial DNA. Exp Physiol, 2003, 88: 41-56

68 Clayton D A. Replication and transcription of vertebrate mitochondrial DNA. Annu Rev Cell Biol, 1991, 7: 453-478

69 Lu J, Sharma L K, Bai Y. Implications of mitochondrial DNA mutations and mitochondrial dysfunction in tumorigenesis. Cell Res, 2009, 19: $802-815$

70 Lisa M. Impact of disease-related mitochondrial mutations on tRNA structure and function. Trends Biochem Sci, 2003, 28: 605-611

71 Villegas J, Zárraga A M, Muller I, et al. A novel chimeric mitochondrial RNA localized in the nucleus of mouse sperm. DNA Cell Biol, 2000, 19: 579-588

72 Villegas J, Müller I, Arredondo J, et al. A putative RNA editing from $\mathrm{U}$ to $\mathrm{C}$ in a mouse mitochondrial transcript. Nucleic Acids Res, 2002, 30: 1895-1901

73 Villegas J, Burzio V, Villota C, et al. Expression of a novel noncoding mitochondrial RNA in human proliferating cells. Nucleic Acids Res, 2007, 35: 7336-7347

74 Burzio V A, Villota C, Villegas J, et al. Expression of a family of noncoding mitochondrial RNAs distinguishes normal from cancer cells. Proc Natl Acad Sci USA, 2009, 106: 9430-9434

75 Castelnuovo M, Massone S, Tasso R, et al. An Alu-like RNA promotes cell differentiation and reduces malignancy of human neuroblastoma cells. FASEB J, 2010, 24: 4033-4046

76 Christov C P, Trivier E, Krude T. Noncoding human Y RNAs are overexpressed in tumours and required for cell proliferation. $\mathrm{Br} \mathrm{J}$ Cancer, 2008, 98: 981-988

77 Gee H E, Buffa F M, Camps C, et al. The small-nucleolar RNAs commonly used for microRNA normalisation correlate with tumour pathology and prognosis. Br J Cancer, 2011, 104: 1168-1177

Open Access This article is distributed under the terms of the Creative Commons Attribution License which permits any use, distribution, and reproduction in any medium, provided the original author(s) and source are credited. 